\title{
Flora do Rio de Janeiro: Molluginaceae
}

\author{
Flora of Rio de Janeiro: Molluginaceae
}

Ricardo Sousa Couto ${ }^{1,3}$ \& Rosana Conrado Lopes ${ }^{2}$

\begin{abstract}
Resumo
O presente estudo tem como objetivo contribuir para o conhecimento das espécies de Molluginaceae ocorrentes no estado do Rio de Janeiro. O trabalho tem como base a análise de coleções depositadas em herbários e coletas de campo, além da consulta de dados de literatura. Foram registrados dois gêneros com uma espécie cada: Glinus e Mollugo. São apresentadas descrições, comentários, além de dados sobre hábitat e distribuição geográfica das espécies.
\end{abstract}

Palavras-chave: flora, Glinus, Mollugo, Sudeste, taxonomia.

\begin{abstract}
This work aims to contribute to the Flora of Molluginaceae from Rio de Janeiro state. It is based on the analysis of herbarium specimens and fieldwork, as well as on the relevant literature. Two genera and three species were recorded: Glinus and Mollugo. Descriptions and comments, besides data on habitat and geographical distribution of the species are provided.
\end{abstract}

Key words: flora, Glinus, Mollugo, Southeast, taxonomy.

\section{Molluginaceae}

Ervas ou subarbustos, anuais ou perenes, carnosas ou não, glabras ou pilosas. Folhas alternas, opostas ou verticiladas, com ou sem bainha; lâmina inteira, simples. Inflorescências cimosas, raro racemosas, plurifloras ou unifloras, axilares, raro terminais. Flores bissexuadas, hipóginas ou ligeiramente períginas; cálice persistente, imbricado, frequentemente gamossépalo, geralmente 5 lóbulos; corola reduzida ou ausente; estames (2)5-8 (ou muitos), livres ou concrescidos na base, estaminódios ausentes ou pequenos e petalóides; ovário súpero, geralmente sincárpico, com 2-5 carpelos, plurilocular, número de estiletes ou estigmas igual ao de lóculos, pluriovular ou solitários. Fruto cápsula circuncisa ou loculicida, raramente indeiscente; sementes por vezes com uma carúncula, embrião curvado em torno de um perisperma amiláceo, endosperma verdadeiro ausente.

Molluginaceae pertence à ordem Caryophyllales, possui cerca de 14 gêneros e 120 espécies distribuídas principalmente em regiões tropicais e subtropicais (Dequan \& Hartmann 2003). No Brasil ocorrem três gêneros (Mollugo L., Glinus L. e Glischrothamnus Pilg.), todos com uma espécie para o país (BGF 2015). No estado do Rio de Janeiro, Molluginaceae está representada por duas espécies, Mollugo verticilata L. e Glinus radiatus (Ruiz \& Pav.) Rohrb. A família não apresenta registro de uso para alimentação nem espécies cultivadas de importância econômica.

\section{Chave para identificação dos gêneros}

1. Folhas elípticas, orbiculares a largo-espatuladas, tomentosas; sépalas cuculadas; sementes com arilo.

1. Glinus

1'. Folhas lineares ou lanceoladas, glabras; sépalas não cuculadas, sementes sem arilo. 2. Mollugo

\footnotetext{
${ }^{1}$ Universidade Federal do Rio de Janeiro, Prog. Pós-graduação em Botânica, Depto. Botânica, Museu Nacional, Quinta da Boa Vista s/n, São Cristóvão, 20940-040, Rio de Janeiro, RJ, Brasil.

${ }^{2}$ Universidade Federal do Rio de Janeiro, IB-CCS, Depto. Botânica, Ilha do Fundão, 21941-590, Rio de Janeiro, RJ, Brasil. rosana@biologia.ufrj.br

${ }^{3}$ Autor para correspondência: rsscouto@gmail.com
} 
Glinus L.

Ervas prostradas ou eretas, muito ramificadas, pilosas, indumento estrelado ou simples. Folhas alternas ou verticiladas, subsésseis, geralmente desiguais; lâmina elíptica, orbicular a largoespatulada, sem bainha. Inflorescência em dicásio axilar ou glomérulo. Flores subsésseis a sésseis; sépalas 5, livres, cuculadas; estames (3-)5(-20), livres ou concrescidos quando numerosos; ovário 3(-5)-carpelar, 3(-5)-locular, pluriovular; estiletes curtos, estigmas 3-5, curvados, papilares. Cápsula loculicida, 3(-5)-valvar, ovada a elíptica; sementes numerosas, reniformes, lisas ou ornamentadas, com apêndice filiforme, arilo e carúncula presentes.

Segundo Dequan \& Hartmann (2003) Glinus é composto por cerca de 10 espécies, ocorrendo em regiões tropicais e subtropicais de ambos os hemisférios, estendendo ainda para poucas regiões temperadas mais quentes. No Brasil, assim como no Estado do Rio de Janeiro, somente uma espécie é encontrada.

Glinus radiatus (Ruiz \& Pav.) Rohrb. in Mart., Fl. bras. 14(2): 238. 1872.

Mollugo radiata Ruiz \& Pav., Flora Peruviana, et Chilensis 1: 48. 1798.

Ervas anuais. Folhas membranáceas, verticiladas; lâmina $1,5-2,5 \times 0,9-1,6 \mathrm{~cm}$, suborbicular a largo-espatulada, ápice obtuso, base aguda, pilosas em ambas as faces. Inflorescência em glomérulo axilar. Flores alvas a esverdeadas; sésseis; sépalas persistentes 5 , lóbulos elípticos 2-4 $\mathrm{mm}$, côncavas, pilosas no dorso, ápice mucronado, base atenuada; estames $3-5$, livres, filetes filiformes, 1,7-3,5 mm; ovário elipsóide, 1,5 × 0,5 $\mathrm{mm}$, estiletes 3, 0,5-1 mm, divergentes. Cápsula loculicida, $4-5 \times 2-3 \mathrm{~mm}$, elíptica; sementes 20-30, 0,3 mm diâm., ferrugíneas, reniformes, arilo carnoso.

Material examinado: Itatiaia, planalto, V.1926, fl. e fr., A.J. de Sampaio 4801 (R).

Material adicional examinado: GOIÁS: Mun. Alto Paraíso, Vale da Lua, 17.VII.2000, fl. e fr., J. Fontella et al. $3412(\mathrm{R})$.

A espécie distribui-se pelas regiões tropicais das Américas, ocorrendo naturalmente em regiões próximas ao mar, lagos e rios (Segada-Vianna et al. 1967). No Brasil possui registros para os estados do Acre, Amazonas, Goiás, Maranhão, Mato Grosso, Minas Gerais, Pará, Paraíba, Pernambuco, Rio Grande do Sul, Rondônia, Roraima e São Paulo (BGF 2015), sendo bastante rara no Rio de Janeiro (P4). Segundo Segadas-Vianna et al. (1967) a espécie é citada por Saint Hilaire em 1829 para a região de Campos dos Goytacazes. Porém, não foram encontrados registros desta espécie para a região, nem tão pouco a citação de Saint Hilaire. Coletada com flores e frutos durante o mês de maio. A espécie é conhecida em todo o Brasil como beldroega ou beldroeguinha, não sendo reportados usos para a espécie no Brasil.

\section{Mollugo L.}

Ervas prostradas ou eretas, de ramificação difusa ou falsamente dicotômica, glabras. Folhas subsésseis, verticiladas, estipulas caducas; lâmina lineares ou lanceoladas, sem bainha. Inflorescência em dicásio ou pleiocásio, raro em umbelas. Flores pediceladas; sépalas 5 , raro 4 , livres, geralmente de margem escariosa transparentes, imbricadas; estames 3-10, livres, ou inseridos no cálice; ovário 3-5-locular, pluriovular; estiletes 3-5, lineares, curtos. Cápsula loculicida, 3(-5)-valvar, globosa, membranácea; sementes numerosas, reniformes ou semicirculares, lisas ou ornamentadas, sem arilo ou carúncula.

Segundo Dequan \& Hartmann (2003) Mollugo é composto por 35 espécies, ocorrendo em regiões tropicais e subtropicais de ambos os hemisférios, estendendo ainda para regiões temperadas mais quentes da Europa, Ásia e América do Norte. No Brasil, assim como no estado do Rio de Janeiro, somente uma espécie nativa é encontrada, havendo também a ocorrência de Mollugo pentaphylla L., espécie exótica naturalizada com ocorrência para o estado do Amazonas.

Mollugo verticillata L., Sp. Pl. 1: 89. 1753.

Ervas anuais, eretas, de ramificação difusa. Folhas membranáceas, verticiladas, agrupadas de 3-6; lâmina 3-30 × 1-9 mm, linear-espatulada a oblongo-linear, ápice obtuso, base aguda. Inflorescência em dicásio, axilar, 3-10 flora. Flores alvas a esverdeadas; pedicelo $3-5 \mathrm{~mm}$; sépalas persistentes 5, lóbulos elípticos ou oblongos 2-3 $\times 1-1,5 \mathrm{~mm}$, ápice e base obtusos; estames 3(-5), livres, inseridos no cálice, filetes filiformes, 2 $\mathrm{mm}$; ovário ovado, $1-1,5 \times 0,5 \mathrm{~mm}$, estiletes 3 , $0,5-0,8 \mathrm{~mm}$, eretos ou levemente divergentes. Cápsula loculicida, 2-2,5 × 1,2-1,6 mm, elíptica, 3-sulcada; sementes 8-30, 0,5 mm diâm., marrons, estriadas, reniformes.

Material selecionado: Araruama, Apa da Massambaba, 10.III.1998, fl. e fr., L. Emygdio et al. 6201 (R); Armação dos Búzios, Praia Brava, 28.X.10, fl. e fr., R.S. Couto 
454 (RFA); Arraial do Cabo, Praia Pernambucana, 3.XII.2007, fl. e fr., D. Araujo 11058 (GUA); Barra Mansa, caminho para Ribeirão de São Joaquim, 21.III.1989, fl., P.E. Diaz et al. 4 (GUA); Cabo Frio, Km 21,5 da estrada S. Pedro da Aldeia, fl. e fr., $F$. Palmieri (RB 182297); Campos dos Goytacazes, Praia Boa Vista, 12.VII.1978, fl., D. Araujo \& N.C. Maciel 2167 (GUA); Carapebus, entre Lagoa de Carapebus e Lagoa Paulista, 28-30.XI.1995, fl., A. Costa et al. 567 (R); Casimiro de Abreu, Barra de São João, 24.V.1964, fl. e fr., A. Castellanos 24957 (GUA); Macaé, Restinga em moitas entre as lagoas Comprida e Carapeba, fl., C. Farney 3517 (RB); Maricá, 28.VII.1971, fl. e fr., L. Monteiro (GUA 8344); Niterói, Restinga da Praia de Piratininga, 27.X.1963, fl. e fr., G.F.J. Pabst (HB 28123); Nova Friburgo, Conego, pedra do Imperador, fl., R. Mello-Silva 2628 (RB); Petrópolis, 12.XII.1984, fl., V.F. Ferreira 3855 (GUA); Porciúncula, Pedra da Elefantina, 9.I.1984, fl. e fr., J.P.P. Carauta et al. 4533 (GUA); Quissamã, PNRJ, 1 km da entrada do Parque, 31.VIII.2005, fl. e fr., M.C. de Oliveira 1228 (R); Resende, Mauá, 16.I.1887, fl. e fr., Schwacke (R 58926); Rio das Ostras, Restinga da Praia Virgem, 14.XII.1999, fl., H. do N. Braga 687 (R); Rio de Janeiro, Ilha do Governador, próximo ao Jockey Club, 7.IX.1960, fl. e fr., G.F.J. Pabst 5422 (HB); São João da Barra, cerca de $10 \mathrm{~km}$ da cidade, 23.III.1982, fl. e fr., E.S.F. da Rocha et al. 860 (GUA); Saquarema, Reserva Ecol. Est. de Massambaba área entre cordões, 26.X.1993, fl. e fr., M. Jalu 113 (RB); Teresópolis, 6.IV.1917, f1., A.J. de Sampaio 2161 (R).

A espécie distribui-se pelos trópicos e subtrópicos em quase todo o mundo, ocorrendo naturalmente em regiões próximas ao mar, lagos e rios. Como exótica ocorre geralmente em pastagens (Dequan \& Hartmann 2003). No Brasil ocorre em quase todos os estados do país (BGF 2015), apresentando distribuição descontinua e sendo pouco frequente. No Rio de Janeiro ocorre em B27, J36, L35, M33, N31, O19, O23, O29, P5, P17, P28, Q7, Q27, R27, S28, T16, T17, T20, T23, T24, T27. Segundo Reitz (1984) é encontrada em locais expostos ao sol, na vegetação herbácea de restinga, em margens de rios e em regiões rurais. Em países como Estados Unidos, Canadá e algumas regiões da Europa a espécie é frequente em áreas urbanas, adotando um comportamento invasor (SegadasVianna et al. 1967). Coletada com flores e frutos durante todo o ano. A espécie é conhecida em todo o Brasil como Molugo, Cabelo-de-guia, Capim- tapete e Mulungo, sendo considerada como planta daninha em diversos cultivos pelo Brasil (e.g., Maciel et al. 2010; Azevedo et al. 1999).

\section{Referências}

Azevêdo, C.L.L.; Carvalho, J.E.B.; Lopes, L.C. \& Araújo, A.M. 1999. Levantamento de plantas daninhas na cultura da mandioca (Manihot esculenta, Crantz), em um ecossistema Semi-Árido do estado da Bahia. In: X Congresso Brasileiro de Mandioca, Manaus, AM. Resumos. SBM, Embrapa, Manaus. 51p.

BFG. 2015. Growing knowledge: an overview of seed plant diversity in Brazil. Rodriguésia 66: 1085-1113.

Bogle, A.L. 1970. The genera of Molluginaceae and Aizoaceae in the southeastern United States. J. Arnold Arbor 51: 431-462.

Dequan, L. \& Hartmann, H.E.K. 2003. Molluginaceae. In: Wu Zheng-yi \& Raven, P.H. (eds.). Flora of China. Vol. 5. Science Press, Beijing \& Missouri Botanical Garden Press, St. Louis. Pp. 437-439.

Furlan, A. \& Machado, P.A. 2002. Molluginaceae. In: Wanderley, M.G.L.; Shepherd, G.J. \& Giulietti, A.M. (coords.). Flora Fanerogâmica do estado de São Paulo. Insituto de Botânica, São Paulo. Vol. 2, pp. 187-188.

Maciel, C.D.G.; Poletine, J.P.; Neto, A.M.O.; Guerra, N. \& Justiniano, W. 2010. Levantamento fitossociológico de plantas daninhas em cafezal orgânico. Vol. 69, n. 3. Bragantia, Campinas. Pp. 631-636.

Reitz, R. 1984. Molugináceas. In: Reitz, R. (ed.). Flora Ilustrada Catarinense. Herbário Barbosa Rodrigues, Itajaí. Fasc. Molu: 1-13.

Rohrbach, P. 1872. Molluginaceae. In: Martius, C.F.P.; Eichler, A.G. \& Urban, I. (ed.). Flora brasiliensis. Lipsiae, Frid. Fleischer. Vol. 14, pars 2, pp. 229-244, t. 55.

Segadas-Vianna, F.; Ormond, W. \& Dau, L. 1967. Molluginaceae. In: Segadas-Viana, F.; Ormond, W. \& Dau, L. (ed.). Flora ecológica de restingas do Sudeste do Brasil. Vol. 7. Museu Nacional, Rio de Janeiro. Pp. 1-12.

Short, P.S. 2011. Molluginaceae. In: Short, P.S. \& Cowie, I.D. (eds.). Flora of the Darwin Region. Vol. 1. Northern Territory Herbarium, Department of Natural Resources, Environment, the Arts and Sport, Palmerston. Pp. 1-7.

Teixeira, L. 1959. Aizoaceae da cidade do Rio de Janeiro. Rodriguésia 33-34: 289-298.

Xifreda, C.C. 2012. Catálogo de las plantas vasculares de la Argentina II. Molluginaceae. Instituto de Botánica Darwinion. Disponivel em $<\mathrm{http}$ ://www2.darwin. edu.ar/Publicaciones/CatalogoVascII/CatalogoVascII. asp>. Acesso em 24 abril 2014. 


\section{Lista de exsicatas}

Aguillar 117 (2.1); Alice, M. 27 (2.1); Andrade, A.G. 1595 (2.1); Araujo, D.S.D. 44, 781, 9082, 4818, 11058, 3968, 2167 (2.1); Ascenção, M.R. 3 (2.1); Assupção, J. 15 (2.1); Barreiros, H. de S. s.n. (2.1); Bove, C.P. 1250 (2.1); Brade, A.C. 11055, 12875, s.n. (2.1); Braga, H. do N. 694, 687 (2.1); Carauta, J.P.P. 4533 (2.1); Carvalho, D.A. 70 (2.1); Castellanos, A. 24957, 23567, s.n. (2.1); Cavalcanti, A.C.S. 85, 128, 156 (2.1); Correia 675, 670 (2.1); Costa, A. 567 (2.1); Couto, R.S. 454 (2.1); Demitri, L. 3563 (2.1); Diaz, P.E. 4 (2.1); Diogo, J.C. 840 (2.1); Dublasievicz, M. 6 (2.1); Emygdio, L. 6201 (2.1); Esteves, R.L. 909, 226 (2.1); Esteves, V. s.n. (2.1); Farney, C. 232, 2154, 3517, 4551, 2154 (2.1); Ferreira, V.F. 3855 (2.1); Ferreira, V.F. 401 (2.1); Fevereiro, P.C.A. 48 (2.1); Fonseca, V.S. 196, 109 (2.1); Fontella, J. 3669, 3485, 3568, 3909, 3787, 3792, 3971, 4096, 2285, 2267, 3090, 3142, 3099, 3103 (2.1), 3412 (1.1); Frazão, A. s.n. (2.1); Freire 564 (2.1); Freitas, M.F. 354 (2.1); Gaglianone, M.C. 23 (2.1); Gimarães, E.F. 58, 152 (2.1); Glaziou, A. 15877, 60 (2.1); Ichaso, C.L.F. 183 (2.1); Jalu, M. 113 (2.1); Klein, V.L.G. 208 (2.1); Konno, T. 434 (2.1); Leitman, M. 1 (2.1); Lima 668, 661 (2.1); Lima, H.C. de 680 (2.1); Löfgren, A. 468, 53, 84 (2.1); Machado, O.X. de B. s.n. (2.1); Marques, M.C. 244 (2.1); Marquete, R. 3447 (2.1); Martinelli, G. 2464 (2.1); Martins, V.L.C. 346, 254, 170, s.n., 623 (2.1); Mautone, L. 127, 134 (2.1); Mello-Silva, R. 2628 (2.1); Melo Filho, L. E 1150 (2.1); Montalvo, E.A. s.n. (2.1); Monteiro, L. s.n. (2.1); Nunes, R. 232 (2.1); Occhioni s.n. (2.1); Oliveira, A.S. 3749 (2.1); Oliveira, F. de 434 (2.1); Oliveira, M.C. de 402, 997, 923, 1228 (2.1); Pabst, G.F.J. s.n. (2.1), 5422 (2.1); Palmieri, F. s.n. (2.1); Pedrini, A.G. 263 (2.1); Pedrosa, D.S. 755 (2.1); Pereira, E. 1, 540, 3563 (2.1); Pereira, J.F. 28 (2.1); Pinheiro 622 (2.1); Poland, C. 6687 (2.1); Ribeiro, R. 222 (2.1); Rocha, E.S.F. da 860 (2.1); Rodarte, A.T.A. 227 (2.1); Sampaio, A.J. de 1334, s.n., s.n., s.n., 2749, 2161, 8169, 8159, 8149, 6319 (2.1), 4801 (1.1); Santos, M.G. 322, 536 (2.1); Scheinvar, L. 48 (2.1); Schwacke s.n. (2.1); Segadas-Vianna 4450, 4648, 4652, 4544, I-1429 (2.1); Silva, I.M. de 80 (2.1); Smith, L.B. 6645, 6392 (2.1); Sobrinho, J.P.L. 926 (2.1); Souza, A. s.n., 2105, 2088, 1922, 1677 (2.1); Souza, A.B. de 74 (2.1); Sucre, D. 1003, 911, 1188, 3819 (2.1); Ule, E. 3975 (2.1); Vianna, M.C. 1886 (2.1); Vidal, J. III-1653 (2.1); Vidal, W.N. 283 (2.1); Viegas, A.P. 2429 (2.1); Vieira, M. 69 (2.1). 